\title{
Research on the Quality Comprehensive Evaluation of Computer Software Based on Grey Clustering Method
}

\author{
Jimin Gao \\ Department of Computer Engineering, Shenzhen Polytechnic, Shenzhen,China \\ gaojm@szpt.edu.cn
}

Keywords: Computer software, Software quality, Grey clustering

\begin{abstract}
With the development of information technology, there are an increasingly demands for high-quality software. the software has been applied in various fields of national economy, and the software quality has been emphasized more and more. How to improve the quality of software products has emerged as an important part of the development of the software industry, which receives the industry's attention. Based on the grey system theory, this paper established the grey clustering comprehensive evaluation model of the computer software quality, which can provide a really effective method for quantitative evaluation of software quality.
\end{abstract}

\section{Introduction}

The grey theory thought that the system that the information is fully known is defined as white system, the system that the information is completely unknown is defined as black system, and the system that the information is between the black and white system, namely part of the information is known and part of the information is unknown, is defined as grey system [1]. The quality of computer software is affected by many factors, and it is of incompleteness. However, the central element of gray system theory is to fully use the available information, take the gray system desalination and whitening. Therefore, using the grey system and its related theory and method to evaluate the quality of computer software is in line with objective reality, and its result is more reasonable. This paper applied grey clustering evaluation method to evaluated the computer software which is easy to program and implement [2,3].

\section{The Principle of Grey Clustering Comprehensive Evaluation}

Grey clustering comprehensive evaluation is based on the generation of whitening function of gray number. It is to comprehensively evaluate the clustering objects for the whitening number of different clustering indexes according to several gray induction class [4,5].

Sign I, II, III and so on as clustering objects, $k=1,2, \cdots, K$, namely the computer software need to be evaluated. Sign $1^{\#}, 2^{\#}, 3^{\#}$ and so on as clustering indexes, $j=1,2, \cdots, m$. Sign $1,2,3, \cdots$ as Clustering gray class, $i=1,2, \cdots, n$. Sign $d_{k i}$ as whitening data of the $k$-th cluster objects for the $i$-th cluster indicators.

Grey clustering perform the following steps. Divide the grey class. The process of gray clustering the computer software quality is to divide the indicators of computer software quality evaluation standard, such as software reliability, maintainability, functionality, usability, efficiency, etc., into several items. Sign them as $U, U=\left\{U_{1}, U_{2}, \cdots, U_{m}\right\}$. Divide each indicator into some grey classes according to quality levels, sign as $V, V=\left\{V_{1}, V_{2}, \cdots, V_{n}\right\}$. The grey class division is shown in Table 1 . In this table, $r_{i j}$ represents the $i$-th gray class's dimensionless value of the $j$-th indicator.

Get the clustering whitening sample data $d_{k j}$. According to the different combination of $k$ and $j$, the following sample matrix can be obtained. 
Table 1. Grey class division

\begin{tabular}{cccccc}
\hline Grey class & Indicator 1 & Indicator 2 & Indicator 3 & $\cdots$ & Indicator $m$ \\
\hline$V_{1}$ & $r_{11}$ & $r_{12}$ & $r_{13}$ & $\cdots$ & $r_{1 \mathrm{~m}}$ \\
$V_{2}$ & $r_{21}$ & $r_{22}$ & $r_{23}$ & $\cdots$ & $r_{2 \mathrm{~m}}$ \\
$\cdots$ & $\cdots$ & $\cdots$ & $\cdots$ & $\cdots$ & $\cdots$ \\
$V_{\mathrm{n}}$ & $r_{\mathrm{n} 1}$ & $r_{\mathrm{n} 2}$ & $r_{\mathrm{n} 3}$ & $\cdots$ & $r_{\mathrm{nm}}$ \\
\hline
\end{tabular}

$$
D=\left(\begin{array}{ccccc}
d_{11} & d_{12} & d_{13} & \cdots & d_{1 m} \\
d_{21} & d_{22} & d_{23} & \cdots & d_{2 m} \\
d_{31} & d_{32} & d_{33} & \cdots & d_{3 m} \\
\vdots & \vdots & \vdots & \ddots & \vdots \\
d_{K 1} & d_{K 2} & d_{K 3} & \cdots & d_{K m}
\end{array}\right)
$$

For example, $d_{12}$ represents the second indicator's point of the first evaluated software.

Determine the whitening weight function of grey class. The level for each index is a change in range, which is a grey number. The grey number is not a definite number, but a range. The grey number related levels corresponding to any whitening number in this interval are not exactly the same. Thus, the whitening function is used to designate. There are three basic forms of whitening function. Figure 1 shows the first class whitening function diagram. $\lambda_{1 j}$ represents the threshold of the first class whitening function of the $\mathrm{j}$-th indicator. $\lambda_{2 j}$ represents the threshold of the second class whitening function of the $\mathrm{j}$-th indicator. Figure 2 shows the whitening function diagram from the second class to the $n$-1-th class. $\lambda_{(i-1) j}$ represents the threshold of the $i$-1-th class whitening function of the $j$-th indicator. $\lambda_{i j}$ represents the threshold of the i-th class whitening function of the $\mathrm{j}$-th indicator. $\lambda_{(i+1) j}$ represents the threshold of the $i+1$-th class whitening function of the $\mathrm{j}$-th indicator. Figure 3 shows the n-th class whitening function diagram. $\lambda_{(n-1) j}$ represents the threshold of the $n$-1-th class whitening function of the $\mathrm{j}$-th indicator. $\lambda_{n j}$ represents the threshold of the $n$-th class whitening function of the $\mathrm{j}$-th indicator. When the whitening function is $\lambda_{i j}$, the $\mathrm{i}$-th grey class's whitening function of the $\mathrm{j}$-th indicator is the most intimate.

$$
\lambda_{1 j}=r_{1 j} / 2, \quad \lambda_{n j}=2 r_{n j}-\lambda_{(n-1) j}, \quad \lambda_{i j}=\left(r_{i j}+r_{(i-1) j}\right) / 2
$$

Where the level range of the n-th whitening function is $\left\lfloor\lambda_{(n-1) j}, \infty\right]$, which has no central value of the grey class. Their threshold is determined by the principles of the same values between the $n-1$-th class and the $n$-th class.

Sign $f_{i j}\left(d_{k j}\right), i=1,2, \cdots, n, \quad j=1,2, \cdots, m, k=1,2, \cdots, K$, as the whitening function value of the $\mathrm{k}$-th grey class of the $\mathrm{j}$-th indicator.

The forms of the whitening function is shown as below:

(1) Grey class $\otimes \in\left[0, \lambda_{1 j}, \lambda_{2 j}\right\rfloor$, shown in Figure 1.

$$
\left\{\begin{array}{l}
f_{i j}\left(d_{k j}\right)=L_{i j}\left(d_{k j}\right)=\left(\lambda_{2 j}-d_{k j}\right) /\left(\lambda_{2 j}-\lambda_{1 j}\right), \\
f_{i j}\left(d_{k j}\right)=1, \quad d_{i j} \in\left[0, \lambda_{1 j}\right]
\end{array}\right.
$$

(2) Grey class $\otimes \in\left[\lambda_{(i-1) j}, \lambda_{i j}, \lambda_{(i+1) j}\right]$, shown in Figure 2.

$$
\begin{cases}f_{i j}\left(d_{k j}\right)=L_{i j}\left(d_{k j}\right)=\left(d_{k j}-\lambda_{(i-1) j}\right) /\left(\lambda_{i j}-\lambda_{(i-1) j}\right), & d_{k j} \in\left[\lambda_{(i-1) j}, \lambda_{i j}\right] \\ f_{i j}\left(d_{k j}\right)=1, & d_{k j}=\lambda_{i j} \\ f_{i j}\left(d_{k j}\right)=0, & d_{k j} \notin\left[\lambda_{(i-1) j}, \lambda_{(i+1) j}\right] \\ f_{i j}\left(d_{k j}\right)=R_{i j}\left(d_{k j}\right)=\left(\lambda_{(i+1) j}-d_{k j}\right) /\left(\lambda_{(i+1) j}-\lambda_{i j}\right), & d_{i j} \in\left[\lambda_{i j}, \lambda_{(i+1) j}\right]\end{cases}
$$




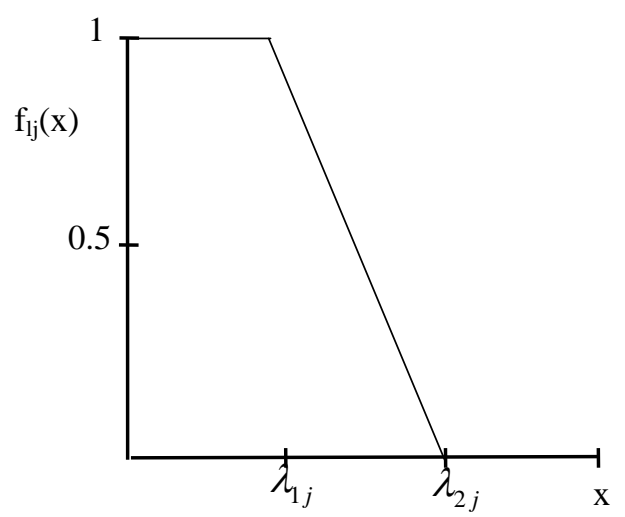

Figure 1 . The first class whitening function

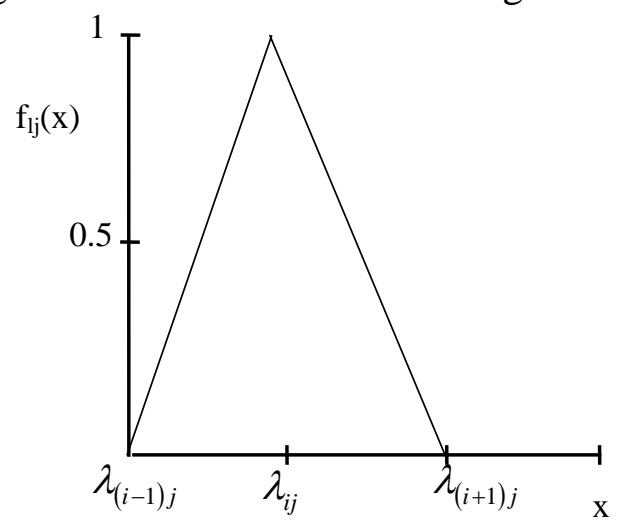

Figure 2. The second class whitening function

(3) Grey class $\otimes \in\left[\lambda_{(n-1) j}, \infty\right]$, shown in Figure 3.

$$
\left\{\begin{array}{l}
f_{i j}\left(d_{k j}\right)=L_{i j}\left(d_{k j}\right)=\left(d_{k j}-\lambda_{(n-1) j}\right) /\left(\lambda_{n j}-\lambda_{(n-1) j}\right), \\
f_{i j}\left(d_{k j}\right)=1, \quad d_{i j} \in\left[\lambda_{n j}, \infty\right]
\end{array}\right.
$$

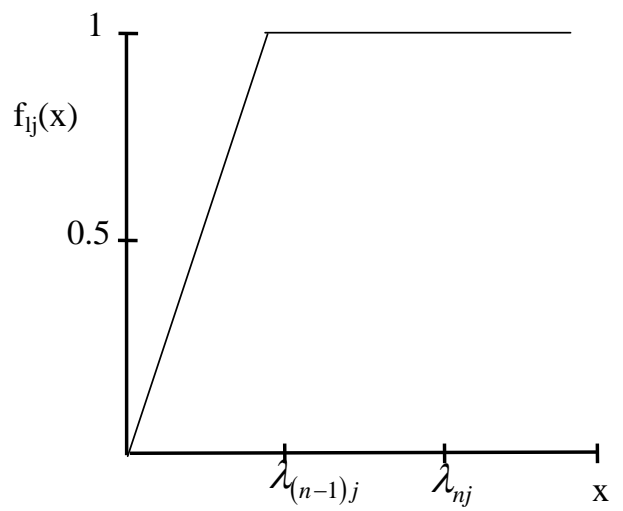

Figure 3. The third class whitening function

Calculate the calibration clustering weight $\eta_{i j}$. Sign $\eta_{i j}$ as the calibration clustering weight of the i-th grey class of the $j$-th indicator. Thus,

$$
\eta_{i j}=\frac{\lambda_{i j}}{\sum_{j=1}^{m} \lambda_{i j}}, j=1,2, \cdots, m
$$

Where $\lambda_{i j}$ represents the threshold.

Calculate the clustering coefficient $\sigma_{i k}$

$$
\sigma_{i k}=\sum_{j=1}^{m} f_{i j}\left(d_{k j}\right) \cdot \eta_{i j}
$$

Where $\sigma_{i k}$ represents the grey clustering coefficient of the i-th grey class for all indicators' k-th 
clustering objects.

Determine the clustering vector $\sigma_{k}$. Determine the clustering vector $\sigma_{k}$ as the following. $\sigma_{i k}=\left(\sigma_{k 1}, \sigma_{k 2}, \cdots, \sigma_{k n}\right), \sigma_{k}$ is the vector of $\sigma_{i k}$.

Comprehensively evaluate based on grey clustering. According to the largest cluster evaluation, that is, if it satisfies the following condition.

$$
\sigma_{i * k}=\max \sigma_{i k}=\max \left(\sigma_{1 k}, \sigma_{2 k}, \cdots, \sigma_{n k}\right)
$$

Called it belongs to cluster $i$.

\section{The Application of Software Quality Evaluation Model Based on Grey Clustering}

Software quality reflects the requirements and needs of those who demand that reflects the user's point of view, according to the definition of software quality characteristics and McCall model of ISO, this paper takes the evaluation from the following six aspects: software reliability $\left(\mathrm{c}_{1}\right)$, maintainability $\left(c_{2}\right)$, functionality $\left(c_{3}\right)$, usability $\left(c_{4}\right)$, efficiency $\left(c_{5}\right)$ and portability $\left(c_{6}\right)[6,7]$.

The evaluation indicators are divided into four level: excellent (I), good (II), middle (III), poor (IV) [8], and the evaluation criteria of these four levels is shown in Table 2.

Table 2. The evaluation criteria

\begin{tabular}{ccccccc}
\hline & $c_{1}$ & $c_{2}$ & $c_{3}$ & $c_{4}$ & $C_{5}$ & $C_{6}$ \\
\hline I & {$[0.85,1)$} & {$[0.80,1)$} & {$[0.51,1)$} & {$[0.75,1)$} & {$[0.60,1)$} & {$[0.81,1)$} \\
II & {$[0.79,0.85)$} & {$[0.60,0.80)$} & {$[0.35,0.51)$} & {$[0.61,0.75)$} & {$[0.45,0.60)$} & {$[0.70,0.81)$} \\
III & {$[0.50,0.79)$} & {$[0.40,0.60)$} & {$[0.20,0.35)$} & {$[0.33,0.61)$} & {$[0.30,0.45)$} & {$[0.50,0.70)$} \\
IV & $(0,0.50)$ & $(0,0.40)$ & $(0,0.20)$ & $(0,0.33)$ & $(0,0.30)$ & $(0,0.50)$ \\
\hline
\end{tabular}

According the indicator level in Table 2, we can divide the grey classes, which is shown in Table 3. The threshold set is shown in Table 4. The evaluation tolerance for gray class criteria is shown in Table 5.

Table 3. The grey class division

\begin{tabular}{ccccccc}
\hline & $c_{1}$ & $c_{2}$ & $c_{3}$ & $c_{4}$ & $c_{5}$ & $c_{6}$ \\
\hline I & {$[0.0,0.50)$} & {$[0.0,0.40)$} & {$[0.0,0.20)$} & {$[0.0,0.33)$} & {$[0.0,0.30)$} & {$[0.0,0.50)$} \\
II & {$[0.50,0.79)$} & {$[0.40,0.60)$} & {$[0.20,0.35)$} & {$[0.33,0.61)$} & {$[0.30,0.45)$} & {$[0.50,0.70)$} \\
III & {$[0.79,0.85)$} & {$[0.60,0.80)$} & {$[0.35,0.51)$} & {$[0.61,0.75)$} & {$[0.45,0.60)$} & {$[0.70,0.81)$} \\
IV & $(0.85,1.00)$ & {$[0.80,1.00)$} & {$[0.51,1.00)$} & {$[0.75,1.00)$} & {$[0.60,1.00)$} & {$[0.81,1.00)$} \\
\hline
\end{tabular}

Table 4. The threshold value

\begin{tabular}{ccccccc}
\hline & $c_{1}$ & $c_{2}$ & $C_{3}$ & $c_{4}$ & $C_{5}$ & $c_{6}$ \\
\hline I & 0.125 & 0.100 & 0.050 & 0.0825 & 0.075 & 0.2500 \\
II & 0.6450 & 0.500 & 0.275 & 0.4700 & 0.375 & 0.6000 \\
III & 0.8200 & 0.700 & 0.430 & 0.6800 & 0.5250 & 0.7550 \\
IV & 0.8725 & 0.800 & 0.5925 & 0.7775 & 0.6255 & 0.8300 \\
\hline
\end{tabular}

Table 5. The evaluated objects

\begin{tabular}{ccccccc}
\hline & $\mathrm{C}_{1}$ & $\mathrm{C}_{2}$ & $\mathrm{C}_{3}$ & $\mathrm{C}_{4}$ & $\mathrm{C}_{5}$ & $\mathrm{C}_{6}$ \\
\hline The evaluated software (1) & 0.90 & 0.79 & 0.65 & 0.80 & 0.61 & 0.82 \\
The evaluated software (2) & 0.60 & 0.50 & 0.30 & 0.40 & 0.40 & 0.60 \\
\hline
\end{tabular}

Use the grey clustering comprehensive evaluation software to calculate the clustering coefficients which are shown in Table 6.

Table 6. The clustering coefficients

\begin{tabular}{lccccc}
\hline & I & II & III & IV & Result \\
\hline The evaluated software (1) & 0 & 0 & 0.087 & 0.913 & Excellent \\
The evaluated software (2) & 0.085 & 0.916 & 0.075 & 0.075 & Medium \\
\hline
\end{tabular}

According to the principle of the largest cluster, the quality of software 1 and software 2 
respectively belongs to the excellent and the middle.

\section{Conclusion}

Comprehensive evaluation method of grey clustering is without critical criterion, and it only need the establishment of evaluation factors based on the quality level of the software to obtain the evaluation results, which can avoid the human subjective and arbitrary. The result of the proposed model is more realistic. Grey clustering evaluation method is simple, practical, versatile, and easy for computer programming. When there are many indicators, multi-grade, and the tolerance evaluation parameter selection is large, it can show its advantage.

\section{References}

[1] Xiaotong Yuan, Huamin Yang. The software quality fuzzy comprehensive evaluation method research [J]. Applied Mechanics and Materials, 2014, 513, 1959-1962.

[2] Yue Feng, Su Zhao-Pin, Lu Yang, Zhang Guo-Fu. Comprehensive evaluation of software quality based on fuzzy soft sets [J]. Systems Engineering and Electronics, 2013, 35(7), 1460-1466.

[3] Pang Qing-hua. Comprehensive evaluation model of human computer interface of software system based on grey theory [J]. Computer Engineering, 2007, 33(18), 59-61.

[4] Yuan Xiao Tong, Yang Hua Min. The software quality fuzzy comprehensive evaluation method research [J]. Applied Mechanics and Materials, 2014, 513, 1959-1962.

[5] Cao Yi, Zhao Bo, Zhang Lifeng. Research and Realization of Software Quality Fuzzy Co[6] Dong Jianli, Shi Ningguo. Research on fuzzy extension synthesis metrics algorithm for software quality [J]. Journal of Software, 2011, 6(11), 2099-2105.

[7] Jianli Dong, Ningguo Shi, Jianzhou Wang. Research and Application on Multi-layer Matter-element Extension Synthesis Evaluation Method for Software Quality [J]. Journal of Software, 2011, 6(9), 1866-1872.

[8] Wang Ren-gang, Ye Zhi-min, Yin Ming-quan. Fuzzy comprehensive evaluation for geological and geomorphic landscape quality of expressways based on analytic hierarchy process [J]. Journal of Chongqing Institute of Technology, 2007, 21(11), 97-72.mprehensive Evaluation [J]. Computer Measurement \& Control, 2013, 21(12), 3306-3308. 